\title{
Equivalences of quadratic APN functions
}

\author{
Satoshi Yoshiara
}

Received: 24 March 2011 / Accepted: 8 August 2011 / Published online: 13 September 2011

(C) Springer Science+Business Media, LLC 2011

\begin{abstract}
The following conjecture due to Y. Edel is affirmatively solved: two quadratic APN (almost perfect nonlinear) functions are CCZ-equivalent if and only if they are extended affine equivalent.
\end{abstract}

Keywords APN functions · Quadratic functions · CCZ-equivalence · Extended affine equivalence

\section{Introduction}

In this paper, we will show the following statement, which was first conjectured by Edel (see Definition 2 and Definition 1 for the exact definitions of notions such as quadratic APN functions and CCZ- and EA-equivalences):

Theorem 1 Let $f$ and $g$ be quadratic APN functions on a finite field $F \cong \mathbf{F}_{2^{n}}$ with $n \geq 2$. Then $f$ is CCZ-equivalent to $g$ if and only if $f$ is EA-equivalent to $g$.

In the recent paper [1], this statement is shown to be true under the assumption that the group of translations is the unique regular elementary abelian 2-subgroup of the automorphism group of a certain code [1, Corollary 4].

In this paper, Theorem 1 is established without any additional assumption. The only use of group theory here is the Sylow theorem and a typical argument on the centralizer of a regular permutation group. Some information, prepared in Sect. 2, about the actions of translations and the description of some graphs is

S. Yoshiara $(\bowtie)$

Department of Mathematics, Tokyo Woman's Christian University, Suginami-ku, Tokyo 167-8585, Japan

e-mail: yoshiara@lab.twcu.ac.jp 
used. The proof is given in Sect. 3. This paper is self-contained except quotations from [2].

Let us mention one possible contribution of Theorem 1 to the current activities in constructing new APN functions. One may use Theorem 1 to simplify the task of showing that an APN function he or she found is new, namely, that it is CCZequivalent neither to any power mapping nor to any member of currently known infinite families, because so far the latter families consist of quadratic functions only.

Now we give an outline of the proof of Theorem 1 with some details. Assume that $f$ and $g$ are quadratic APN functions on a finite field $F \cong \mathbf{F}_{2^{n}}$ with $n \geq 2$, which are CCZ-equivalent. By [2, Proposition 3], this assumption is equivalent to the existence of a graph isomorphism between the graphs $\Gamma_{f}$ and $\Gamma_{g}$ defined on $\mathbf{F}_{2} \oplus F \oplus F$ constructed from these functions (see Definition 3). The existence of certain automorphisms of $\Gamma_{g}$, called "translations" (see (12)) allows us to assume that such an isomorphism, say $\rho$, fixes a point $(0 ; 0,0)$ and a block $(1 ; 0,0)$. For the function $h=f$ or $g$, we denote by $M_{h}$ the stabilizer of $(0 ; 0,0)$ in the automorphism group of the graph $\Gamma_{h}$, and by $T_{h}$ the group of translations of $\Gamma_{h}$ (which is contained in $M_{h}$ ). Applying the Sylow theorem and [2, Lemma 3], we may choose $\rho$ as a linear map on $\mathbf{F}_{2} \oplus F \oplus F$ so that a Sylow 2-subgroup $S_{f}$ of $M_{f}$ containing $T_{f}$ is sent to a Sylow 2-subgroup $S_{g}$ of $M_{g}$ containing $T_{g}$ (see Lemma 10).

We will show that $\rho$ with these properties (called condition (a) in Sect. 3) preserves a subspace $Y=\{(0 ; 0, y) \mid y \in F\}$ of $\mathbf{F}_{2} \oplus F \oplus F$, which is equivalent to the claim that $\rho$ induces an EA-equivalence of $f$ with $g$ (see Lemma 12). We will derive a contradiction assuming that $f$ is not EA-equivalent to $g$, namely, that $\rho$ does not preserve $Y$ (condition (b) in Sect. 3). Based on an observation that the center $Z\left(S_{h}\right)$ of the Sylow subgroup $S_{h}$ lies in $T_{h}$ for both $h=f$ and $g$ (see Lemma 9), we can calculate the centralizer of $Z\left(S_{h}\right)$ on the set of points of $\Gamma_{h}$ (see Lemma 6(3)). If $\left|Z\left(S_{f}\right)\right| \geq 4$, they are equal to the subspace $Y$, whence $Y$ is stabilized by $\rho$. Therefore, we may assume that $\left|Z\left(S_{h}\right)\right|=2(h=f, g)$ (Lemma 13(1)) because a nontrivial 2-group has a nontrivial center. In this case, the image of $Y$ under $\rho$ is one of the two possible subspaces containing the subspace consisting of $\left(0 ; 0, y^{\prime}\right)$, where $y^{\prime}$ ranges over a hyperplane of $F$ (Lemma 7). As we assumed that $\rho$ does not preserve $Y$, the image of $Y$ under $\rho$ is uniquely determined (see Lemma 14). In particular, the values $(x+y)^{\pi}+x^{\pi}+y^{\pi}$ for $x, y \in F$ lie in a one-dimensional subspace spanned by a specific nonzero element $a^{\prime}$ of $F$ (see Lemma 15), where $\pi$ is a permutation on $F$ such that the image of a block $(1 ; x, f(x)+f(0))$ is mapped by $\rho$ to $\left(1 ; x^{\pi}, g\left(x^{\pi}\right)+g(0)\right)$ for every $x \in F$ (see the paragraph after Lemma 10). Then we may introduce a form $\kappa$ on $F$ which vanishes at $(x, y)$ exactly when $B_{f}(x, y)=f(x+y)+f(x)+f(y)+f(0)$ lies in a certain hyperplane $H_{a}$ of $F$ (see (30)). Using (31), we investigate this form to conclude that it is almost the zero form (see Lemma 17). This gives a final contradiction.

The arguments in this proof do not give much information on the structure of automorphism groups of $\Gamma_{f}$ for a quadratic APN function $f$. For example, it seems that they cannot be used to establish the normality of the group of translations in the stabilizer of a point. 


\section{Preliminaries}

In this section, we review some results in [3] on the graph $\Gamma_{f}$ associated with an APN function $f$ on a finite field $\mathbf{F}_{2^{n}}$ with additional remarks in the case where $f$ is quadratic.

Throughout this paper, $F$ denotes a finite field of size $2^{n}$ with $n \geq 2$, unless otherwise stated. We regard $F$ as a vector space of dimension $n$ over $\mathbf{F}_{2}$. Moreover, the following sets $F \oplus F$ and $\mathbf{F}_{2} \oplus F \oplus F$ are regarded as vector spaces of dimensions $2 n$ and $2 n+1$ over $\mathbf{F}_{2}$, respectively:

$$
\begin{aligned}
F \oplus F & :=\{(x, y) \mid x, y \in F\}, \\
\mathbf{F}_{2} \oplus F \oplus F & :=\left\{(\varepsilon ; x, y) \mid \varepsilon \in \mathbf{F}_{2}, x, y \in F\right\} .
\end{aligned}
$$

Let $X$ be one of the following sets: $F, F \oplus F$, and $\mathbf{F}_{2} \oplus F \oplus F$. For a map $\sigma$ on $X$, we usually denote the image of an element $x$ of $X$ under $\sigma$ by $x^{\sigma}$. Thus, the composition $\sigma \tau$ for maps $\sigma$ and $\tau$ on $X$ is defined to be the map on $X$ sending each $x \in X$ to $\left(x^{\sigma}\right)^{\tau}$. If $X=F$ and $\sigma$ is an APN function (see Definition 2 (APN)), we denote by $\sigma(x)$ the image of $x \in X=F$ under $\sigma$, to stress on the fact that $\sigma$ is an APN function. Since we do not consider the composition of APN functions, this exceptional notation may not cause any confusion.

Observe that every $\mathbf{F}_{2}$-linear map $\lambda$ on $F \oplus F$, regarded as a $2 n$-dimensional vector space over $\mathbf{F}_{2}$, is expressed uniquely by the quadruple $(\alpha, \beta, \gamma, \delta)$ of $\mathbf{F}_{2}$-linear maps $\alpha, \beta, \gamma$, and $\delta$ on $F$ such that

$$
(x, y)^{\lambda}=\left(x^{\alpha}+y^{\gamma}, x^{\beta}+y^{\delta}\right)
$$

for every $x, y \in F$. We will denote $\lambda=\lambda(\alpha, \beta, \gamma, \delta)$ if $\lambda$ is expressed in this way. Accordingly, every $\mathbf{F}_{2}$-affine map $\tilde{\lambda}$ on $F \oplus F$ is uniquely expressed as a composition $\lambda(\alpha, \beta, \gamma, \delta) \tau(c, d)$ for some $\mathbf{F}_{2}$-linear maps $\alpha, \beta, \gamma, \delta$ on $F$ and some elements $c, d$ of $F$, where $\tau(c, d)$ is defined by

$$
(x, y)^{\tau(c, d)}:=(x+c, y+d)
$$

for every $x, y \in F$.

With the above convention, we introduce two equivalence relations for functions on $F$.

Definition 1 For a function $f$ on $F$, its graph $G(f)$ is defined to be a subset

$$
G(f):=\left\{\left(x, x^{f}\right) \mid x \in F\right\}
$$

of $F \oplus F$. Let $f$ and $g$ be two functions on $F$.

(CCZ) $f$ is called CCZ-equivalent to $g$ if there is a bijective affine map on $F \oplus F$ sending $G(f)$ to $G(g)$.

(EA) $f$ is called extended affine equivalent (EA-equivalent for short) to $g$ if there is a bijective affine map of the shape $\lambda(\alpha, \beta, 0, \delta) \tau(c, d)($ with $\gamma=0)$ on $F \oplus F$ sending $G(f)$ to $G(g)$. 
We note that the definition of EA-equivalence above coincides with the usual definition of EA-equivalence (see, e.g., [1, Introduction]). For $x, y \in F$, we have

$$
(x, y)^{\lambda(\alpha, \beta, 0, \delta) \tau(c, d)}=\left(x^{\alpha}+c, x^{\beta}+y^{\delta}+d\right) .
$$

Thus, two functions $f$ and $g$ are EA-equivalent if and only if there are $\mathbf{F}_{2}$-linear maps $\alpha, \beta, \delta$ with $\alpha$ and $\delta$ bijective and elements $c, d$ of $F$ such that

$$
g\left(x^{\alpha}+c\right)=f(x)^{\delta}+x^{\beta}+d
$$

for all $x, y \in F$. (Here we denote the images of $f$ and $g$ by $f(x)$ and so on, because we usually use this notation for APN functions $f$ and $g$.) This implies that

$$
g(z)=f\left(z^{\alpha^{-1}}+c^{\alpha^{-1}}\right)^{\delta}+\left(z^{\alpha^{-1} \beta}+c^{\alpha^{-1} \beta}+d\right)
$$

for $z \in F$. Thus, $g$ is the sum of an affine map $\alpha^{-1} \beta \tau\left(c^{\alpha^{-1} \beta}+d\right)$ on $F$ and the composition $\left(\alpha^{-1} \tau\left(c^{\alpha^{-1}}\right)\right) f \delta$, where $\tau(k)$ for $k \in F$ denotes the affine map on $F$ sending each $z \in F$ to $z+k$. This is the usual form adopted as a definition of EAequivalence (see, e.g., [1, Introduction]).

We now introduce some classes of functions on $F$.

Definition 2 Let $f$ be a function on a finite field $F \cong \mathbf{F}_{2^{n}}$.

(APN) $f$ is called almost perfect nonlinear (abbreviated as APN) if

$$
{ }^{\#}\{x \in F \mid f(x+a)+f(x)=b\} \leq 2
$$

for all $a \in F^{\times}:=F \backslash\{0\}$ and $b \in F$.

(Quad) $f$ is called quadratic if

$$
\sum_{\left(x_{a}, x_{b}, x_{c}\right) \in \mathbf{F}_{2}^{3}} f\left(x_{a} a+x_{b} b+x_{c} c\right)=0
$$

for any elements $a, b, c$ of $F$.

We associate with each function $f$ on $F$ a graph $\Gamma_{f}$. We first define, for each function $f$ on $F$, the function $\bar{f}$ by

$$
\bar{f}(x):=f(x)+f(0) \quad(x \in F) .
$$

Definition 3 [3, Definition 4] The set of vertices of $\Gamma_{f}$ is defined to be $\mathbf{F}_{2} \oplus F \oplus F$. A vertex $(\varepsilon ; x, y)$ of $\Gamma_{f}\left(\varepsilon \in \mathbf{F}_{2}, x, y \in F\right)$ is called a point or block according to $\varepsilon=0$ or $\varepsilon=1$. We denote the set of points and blocks by $\mathcal{P}$ and $\mathcal{B}$, respectively. We sometimes identify $\mathcal{P}$ with $F \oplus F$ via the natural identification map sending $(0 ; x, y)$ to $(x, y)$.

$$
\mathcal{P}:=\{(0 ; x, y) \mid x, y \in F\}, \quad \mathcal{B}:=\{(1 ; x, y) \mid x, y \in F\} .
$$

Two vertices $(\varepsilon ; x, y)$ and $\left(\varepsilon^{\prime} ; x^{\prime}, y^{\prime}\right)$ are adjacent in $\Gamma_{f}$ whenever

$$
\varepsilon+\varepsilon^{\prime}=1 \text { and } y+y^{\prime}=\bar{f}\left(x+x^{\prime}\right) .
$$


It is easy to see that the following maps $\iota$ and $\tau(a, b)(a, b \in F)$ are graph automorphisms of $\Gamma_{f}$ for any function $f$ on $F$ :

$$
\begin{aligned}
\iota:(\varepsilon ; x, y) & \mapsto(\varepsilon+1 ; x, y), \\
\tau(a, b):(\varepsilon ; x, y) & \mapsto(\varepsilon ; x+a, y+b) .
\end{aligned}
$$

Observe that $\tau(a, b)$ is a bijective affine map on $\mathbf{F}_{2} \oplus F \oplus F$ stabilizing the set $\mathcal{P}$ of points. Thus, its restriction on $\mathcal{P}$ is a bijective affine map on $\mathcal{P}=F \oplus F$ which coincides with the map $\tau(a, b)$ on $F \oplus F$ defined in (2). Thus, we also denote this restriction on $\mathcal{P}$ by $\tau(a, b)$.

For a vertex $v$ of $\Gamma_{f}$ and a nonnegative integer $i$, we denote by $\left(\Gamma_{f}\right)_{i}(v)$ the set of vertices of $\Gamma_{f}$ at distance $i$ from $v$. When a function $f$ on $F$ is clear from the context, we just denote it by $\Gamma_{i}(v)$, omitting $f$. We also denote by $\Gamma_{\leq i}(v)$ the subset of vertices of $\Gamma_{f}$ consisting of vertices at distance at most $i$ from $v$.

Lemma 1 [3, Proposition 1] A function $f$ on $F$ is an APN function if and only if the graph $\Gamma_{f}$ is the incidence graph of a semibiplane, namely, if it is a connected graph with the following property:

for any distinct points (resp. blocks), there are exactly 0 or 2 blocks (resp. points) adjacent to both of them.

By the definition of adjacency in $\Gamma_{f}$, the set $\Gamma_{1}(\mathbf{0})$ of blocks of $\Gamma_{f}$ adjacent to $\mathbf{0}=(0 ; 0,0)$ consists of the following $2^{n}=|F|$ blocks:

$$
\Gamma_{1}(\mathbf{0})=\{(1 ; x, \bar{f}(x)) \mid x \in F\} .
$$

Furthermore, for an APN function $f$ on $F$, the set of points at distance two from $\mathbf{0}$ consists of the following $2^{n-1}\left(2^{n}-1\right)$ points:

$$
\Gamma_{2}(\mathbf{0})=\{(0 ; x+y, \bar{f}(x)+\bar{f}(y)) \mid x, y \in F, x \neq y\} .
$$

Lemma 2 [3, Proposition 2] Two APN functions $f$ and $g$ on $F$ are CCZ-equivalent if and only if the corresponding graphs $\Gamma_{f}$ and $\Gamma_{g}$ are isomorphic as graphs.

This result corresponds to [1, Theorem 6], but in terms of the graphs $\Gamma_{f}$ and $\Gamma_{g}$. To establish this claim, the following result is important, where a vector $(\varepsilon ; x, y)$ of $\mathbf{F}_{2} \oplus F \oplus F$ is denoted by $(\varepsilon ; x, y)_{h}$ when it is regarded as a vertex of the graph $\Gamma_{h}$ for a function $h=f$ or $g$ on $F$.

Lemma 3 [3, Lemma 3] Assume that $\lambda$ is a graph isomorphism from $\Gamma_{f}$ to $\Gamma_{g}$ sending $(0 ; 0,0)_{f}$ to $(0 ; 0,0)_{g}$. Then $\lambda$ is an $\mathbf{F}_{2}$-linear map on $\mathbf{F}_{2} \oplus F \oplus F$.

Observe that the map $\lambda$ in Lemma 3 sends a point $(0 ; 0,0)_{f}$ of $\Gamma_{f}$ to a point $(0 ; 0,0)_{g}$ of $\Gamma_{g}$, whence $\lambda$ sends the set $\mathcal{P}_{f}$ of points of $\Gamma_{f}$ to the set $\mathcal{P}_{g}$ of points of $\Gamma_{g}$. Since $\mathcal{P}_{f}$ and $\mathcal{P}_{g}$ are identical to $\mathcal{P}$, the map $\lambda$ preserves both $\mathcal{P}$ and $\mathcal{B}=\left(\mathbf{F}_{2} \oplus F \oplus F\right) \backslash \mathcal{P}$. 
The semidirect product $N\langle\iota\rangle$ of $N:=\{\tau(a, b) \mid a, b \in F\}$ with $\langle\iota\rangle$ (see (5) and (6)) is a subgroup of the automorphism group $\operatorname{Aut}\left(\Gamma_{f}\right)$ of graph $\Gamma_{f}$ which acts regularly on the set $\mathbf{F}_{2} \oplus F \oplus F=\mathcal{P} \cup \mathcal{B}$ of vertices. In fact, $\operatorname{Aut}\left(\Gamma_{f}\right)$ has the following structure:

Lemma 4 [3, Proposition 3] The automorphism group Aut $\left(\Gamma_{f}\right)$ of the graph $\Gamma_{f}$ for an APN function $f$ on $F$ has the subgroup $\operatorname{Aut}\left(\Gamma_{f}\right)_{+}$of automorphisms preserving both $\mathcal{P}$ and $\mathcal{B}$ as a normal subgroup of index 2 . The subgroup $\operatorname{Aut}\left(\Gamma_{f}\right)_{+}$is a semidirect product of a normal subgroup $N=\{\tau(a, b) \mid a, b \in F\}$ with the stabilizer $M$ of a point $(0 ; 0,0)$. The subgroup $M$ consists of $\mathbf{F}_{2}$-linear bijections on $\mathbf{F}_{2} \oplus F \oplus F$ preserving $\mathcal{P}$ and $\mathcal{B}$ together with the adjacency of $\Gamma_{f}$.

Observe that the stabilizer $M$ in Lemma 4 acts on $\Gamma_{i}(\mathbf{0})$ for each nonnegative integer $i$, as $M$ fixes the point $\mathbf{0}$ and preserves the distance on $\Gamma_{f}$.

Now we assume that $f$ is a quadratic APN function on $F$. Then the map $B_{f}$ on $F \times F$ defined by

$$
B_{f}(x, y):=\bar{f}(x+y)+\bar{f}(x)+\bar{f}(y)
$$

for $x, y \in F$ is an $\mathbf{F}_{2}$-bilinear form on $F$. Moreover, as $f$ is an APN function, the kernel of the linear map sending $x \in F$ to $B_{f}(a, x)$ coincides with $\{0, a\}$ for each $a \in F^{\times}$. Thus, for each $a \in F^{\times}$,

$$
H_{a}:=\left\{B_{f}(a, x) \mid x \in F\right\}
$$

is a hyperplane of $F$.

Recall that for every hyperplane $H$ of $F$, there is a unique element $\alpha$ of $F^{\times}$such that $H$ is the kernel of the linear form sending $x \in F$ to $\operatorname{Tr}(\alpha x)$, where $\operatorname{Tr}$ denotes the trace function for extension $F / \mathbf{F}_{2}: \operatorname{Tr}(x)=\sum_{i=0}^{n-1} x^{2^{i}}(x \in F)$. Thus, we may introduce a map $\alpha$ on $F^{\times}$by

$$
H_{a}=\{y \in F \mid \operatorname{Tr}(\alpha(a) y)=0\} .
$$

Next we state a result on hyperplanes $H_{a}\left(a \in F^{*}\right)$ above, which is used in the last part of the proof of Theorem 1. This result is shown, e.g., in [2, Proposition 2.2]. There, the ambient space of the dual hyperoval $\mathcal{S}^{n}[f]$ associated with a quadratic APN function $f$ is shown to be $F \oplus F$. By definition, this subspace consists of vectors $(x, y)$ where $x$ ranges over $F$ and $y$ ranges over the subspace of $F$ spanned by all hyperplanes $H_{b}$ for $b \in F^{\times}$. Thus, this implies that:

Lemma 5 For a quadratic APN function $f$ on $F$, the hyperplanes $H_{b}=\left\{B_{f}(b, x) \mid\right.$ $x \in F\}$ of $F$ for all $b \in F^{\times} \operatorname{span} F$.

For a quadratic APN function $f$ on $F$, we can verify that the following $\mathbf{F}_{2}$-linear map $t_{a}$ for every $a \in F$ is an automorphism of $\Gamma_{f}$ belonging to the stabilizer $M$ of $(0 ; 0,0)$ (and so preserving both $\mathcal{P}$ and $\mathcal{B})$ :

$$
(\varepsilon ; x, y)^{t_{a}}:=\varepsilon(1 ; a, \bar{f}(a))+\left(0 ; x, y+B_{f}(a, x)\right)
$$


for $\varepsilon \in \mathbf{F}_{2}$ and $x, y \in F$. We call $t_{a}$ the translation with respect to $a \in F$. We define $T$ to be the subgroup of $M$ consisting of all translations,

$$
T:=\left\{t_{a} \mid a \in F\right\} .
$$

Since $t_{a} t_{b}=t_{a+b}$ for $a, b \in F, T$ is an elementary abelian group of order $2^{n}=|F|$.

We collect information on the actions of translations on the vertices of $\Gamma_{f}$.

Lemma 6 If $f$ is a quadratic APN function on $F$, the following statements hold for every nonidentity translation $t_{a}\left(a \in F^{\times}\right)$:

(1) The translation $t_{a}$ does not fix any block of $\Gamma_{f}$. In particular, the group $T$ of translations acts regularly on the set $\Gamma_{1}(\mathbf{0})$ of blocks adjacent to $\mathbf{0}$.

(2) The commutator space $\left[\mathcal{P}, t_{a}\right]:=\left\{\mathbf{x}+\mathbf{x}^{t_{a}} \mid \mathbf{x} \in \mathcal{P}\right\}$ of $t_{a}$ on $\mathcal{P}$ is given as

$$
\left[\mathcal{P}, t_{a}\right]=\left\{\left(0 ; 0, B_{f}(a, x)\right) \mid x \in F\right\}=\left\{(0 ; 0, y) \mid y \in H_{a}\right\} .
$$

(3) The centralizer $C_{\mathcal{P}}\left(t_{a}\right):=\left\{\mathbf{x} \in \mathcal{P} \mid \mathbf{x}^{t_{a}}=\mathbf{x}\right\}$ of $t_{a}$ on $\mathcal{P}$ is given as

$$
C_{\mathcal{P}}\left(t_{a}\right)=\{(0 ; a, y),(0 ; 0, y) \mid y \in F\},
$$

which intersects $\Gamma_{2}(\mathbf{0})$ at

$$
C_{\mathcal{P}}\left(t_{a}\right) \cap \Gamma_{2}(\mathbf{0})=\left\{(0 ; a, \bar{f}(a)+y) \mid y \in H_{a}\right\} .
$$

Proof (1) From (12) we have

$$
\begin{aligned}
(1 ; x, y)^{t_{a}} & =\left(1 ; x+a, y+\bar{f}(a)+B_{f}(a, x)\right) \\
& =(1 ; x+a, \bar{f}(x+a)+\bar{f}(x)+y)
\end{aligned}
$$

for $x, y \in F$, as $B_{f}(a, x)=\bar{f}(a+x)+\bar{f}(a)+\bar{f}(x)$. Thus, $t_{a}\left(a \in F^{\times}\right)$does not fix any block of $\Gamma_{f}$, and $T$ acts regularly on $\Gamma_{1}(\mathbf{0})=\{(1 ; x, \bar{f}(x)) \mid x \in F\}$ (see (7)).

(2), (3) Fix $a \in F^{\times}$. From (12) we have

$$
(0 ; x, y)^{t_{a}}=\left(0 ; x, y+B_{f}(a, x)\right)
$$

for $x, y \in F$. Claim (2) follows. We also have $(0 ; x, y)^{t_{a}}=(0 ; x, y)$ if and only if $B_{f}(a, x)=0$, which is equivalent to the condition that $x=0$ or $x=a$. This implies (15). Then Claim (3) follows from the description of $\Gamma_{2}(\mathbf{0})$ (see (8)).

Lemma 7 For a nonidentity translation $t_{a}\left(a \in F^{\times}\right)$, there are exactly two subspaces $X$ of $\mathcal{P}$ of dimension $n$ with the following properties:

(i) $\left[\mathcal{P}, t_{a}\right] \subset X \subset C_{\mathcal{P}}\left(t_{a}\right)$, and

(ii) $X \cap \Gamma_{2}(\mathbf{0})=\emptyset$.

In fact, $X$ is one of the following subspaces $Y$ and $Y(a)$, where $c$ is a fixed element of $F$ not contained in the hyperplane $H_{a}$ (see (10)) of $F$ and $\varepsilon=\operatorname{Tr}(\alpha(a) \bar{f}(a))$, which is equal to 0 or 1 according as $\bar{f}(a) \in H_{a}$ or not (see (11) for the definition of $\left.\alpha(a)\right)$ :

$$
Y:=\{(0 ; 0, y) \mid y \in F\}
$$


and

$$
Y(a):=\left\{(0 ; a,(\varepsilon+1) c+y),(0 ; 0, y) \mid y \in H_{a}\right\} .
$$

Proof From Lemma 6(2)(3) we have $C_{\mathcal{P}}\left(t_{a}\right)=\{(0 ; a, y),(0 ; 0, y) \mid y \in F\}$ and $\left[\mathcal{P}, t_{a}\right]=\left\{(0 ; 0, y) \mid y \in H_{a}\right\}$. Fix an element $c$ in $F \backslash H_{a}$. Then the factor group $C_{\mathcal{P}}\left(t_{a}\right) /\left[\mathcal{P}, t_{a}\right]$ consists of $(0 ; 0, c)+\left[\mathcal{P}, t_{a}\right],(0 ; a, 0)+\left[\mathcal{P}, t_{a}\right],(0 ; a, c)+\left[\mathcal{P}, t_{a}\right]$ together with the trivial coset. Since $X /\left[\mathcal{P}, t_{a}\right]$ is a one-dimensional subspace of $C_{\mathcal{P}}\left(t_{a}\right) /\left[\mathcal{P}, t_{a}\right]$, the subspace $X$ coincides with $Y:=\left\langle(0 ; 0, c),\left[\mathcal{P}, t_{a}\right]\right\rangle, Y_{1}:=$ $\left\langle(0 ; a, 0),\left[\mathcal{P}, t_{a}\right]\right\rangle$, or $Y_{2}:=\left\langle(0 ; a, c),\left[\mathcal{P}, t_{a}\right]\right\rangle$.

Observe that $Y=\left\langle(0 ; 0, c),\left[\mathcal{P}, t_{a}\right]\right\rangle=\{(0 ; 0, y) \mid y \in F\}$ does not contain any point of $\Gamma_{2}(\mathbf{0})$ by (8). Thus, $Y$ is one of the candidates for $X$.

Since $Y_{1} \backslash\left[\mathcal{P}, t_{a}\right]=\left\{(0 ; a, y) \mid y \in H_{a}\right\}$ and $Y_{2} \backslash\left[\mathcal{P}, t_{a}\right]=\left\{\left(0 ; a, y^{\prime}\right) \mid y^{\prime} \in F \backslash\right.$ $H_{a}$ \}, any point of $\left(Y_{1} \cup Y_{2}\right) \backslash\left[\mathcal{P}, t_{a}\right]$ is of the shape $(0 ; a, y)$ for some $y \in F$. It is contained in $\Gamma_{2}(\mathbf{0})$ if and only if $y=\bar{f}(x+a)+\bar{f}(x)=\bar{f}(a)+B_{f}(x, a)$ for some $x \in F$ from (8). Thus, $Y_{1} \cap \Gamma_{2}(\mathbf{0}) \neq \emptyset$ (resp. $Y_{2} \cap \Gamma_{2}(\mathbf{0}) \neq \emptyset$ ) if and only if $Y_{1}$ (resp. $\left.Y_{2}\right)$ contains a point $(0 ; a, \bar{f}(a))$, which is equivalent to $\bar{f}(a) \in H_{a}$ (resp. $\left.\bar{f}(a) \notin H_{a}\right)$. Furthermore, in this case, $Y_{2} \cap \Gamma_{2}(\mathbf{0})=\emptyset$ (resp. $\left.Y_{1} \cap \Gamma_{2}(\mathbf{0})=\emptyset\right)$. Thus, the second candidate for $X$ is either $Y_{2}$ or $Y_{1}$ according as $\bar{f}(a) \in H_{a}$ or not. Summarizing, the second candidate for $X$ is given as $Y(a):=\left\{(0 ; a,(1+\varepsilon) c+y),(0 ; 0, y) \mid y \in H_{a}\right\}$, where $\varepsilon=\operatorname{Tr}(\alpha(a) \bar{f}(a))$.

We give a remark on the action of $M$, the stabilizer of a point $\mathbf{0}=(0 ; 0,0)$ in $\operatorname{Aut}\left(\Gamma_{f}\right)$ (see Lemma 4), on the set $\Gamma_{1}(\mathbf{0})$ of blocks adjacent to $\mathbf{0 .}$

Lemma $8 M$ acts faithfully on the set $\Gamma_{1}(\mathbf{0})$ of blocks adjacent to $\mathbf{0}=(0 ; 0,0)$.

Proof Let $K$ be a subgroup of $M$ acting trivially on $\Gamma_{1}(\mathbf{0})$. Take any positive integer $i$ with $i \geq 2$, and let $v$ be any vertex of $\Gamma_{i}(\mathbf{0})$. Since $\Gamma_{f}$ is connected, there is a vertex $w$ of $\Gamma_{i-2}(\mathbf{0})$ at distance 2 from $v$. Then there are exactly two vertices $B$ and $B^{\prime}$ of $\Gamma_{i-1}(\mathbf{0})$ adjacent to both $v$ and $w$, because $\Gamma_{f}$ is the incidence graph of a semibiplane. Observe that $v$ is the unique vertex in $\Gamma_{i}(\mathbf{0})$ adjacent to both $B$ and $B^{\prime}$ (which are both in $\Gamma_{i-1}(\mathbf{0}) \cap \Gamma_{1}(w)$ ). Thus, if $K$ fixes all vertices in $\Gamma_{\leq i-1}(\mathbf{0})$, then $K$ fixes $v$ as well. Namely, $K$ fixes all vertices in $\Gamma_{\leq i}(\mathbf{0})$. Thus, starting with the assumption that $K$ fixes all vertices in $\Gamma_{\leq 1}(\mathbf{0})$, we conclude that $K$ fixes all vertices in $\Gamma_{f}$, whence $K=1$.

The previous lemma poses some restriction on the center of a Sylow 2-subgroup of $M$ containing $T$, the group of translations (see Lemma 4).

Lemma 9 Let $S$ be a Sylow 2-subgroup of $M$ containing $T$. Then the centralizer $C_{S}(T)$ of $T$ in $S$ coincides with $T$. In particular, the center $Z(S)$ of $S$ is a subgroup of $T$.

Proof Since $S$ fixes the point $\mathbf{0}=(0 ; 0,0)$, it acts on the set $\Gamma_{1}(\mathbf{0})$ of blocks adjacent to $\mathbf{0}$. By Lemma $6(1)$, the group $T$ of translations acts regularly on $\Gamma_{1}(\mathbf{0})=$ $\{(1 ; x, \bar{f}(x)) \mid x \in F\}$ (see (7)). Thus, we have $S=T S_{B}$, where $S_{B}$ denotes the 
stabilizer of a block $B=(1 ; 0,0)$ in $\Gamma_{1}(\mathbf{0})$. Since $T$ is an abelian group, we have $C_{S}(T)=T C_{S_{B}}(T)$, where $C_{S_{B}}(T)$ is the centralizer of $T$ in $S_{B}$. Take any element $\sigma$ of $C_{S_{B}}(T)$. Since $\sigma t_{a}=t_{a} \sigma$ for any $a \in F$, we have

$$
(1 ; a, \bar{f}(a))=B^{t_{a}}=B^{\sigma t_{a}}=B^{t_{a} \sigma}=(1 ; a, \bar{f}(a))^{\sigma}
$$

for all $a \in F$, by (17). Thus, $\sigma$ fixes all the blocks in $\Gamma_{1}(\mathbf{0})$. Hence, $\sigma$ is the identity on $\Gamma_{f}$ by Lemma 8 . Then $C_{S_{B}}(T)=1$ and $C_{S}(T)=T$.

\section{Proof of Theorem 1}

Let $f$ and $g$ be quadratic APN functions on a field $F \cong \mathbf{F}_{2^{n}}$. Assume that $f$ is CCZequivalent to $g$. Then there is a graph isomorphism $\rho$ from $\Gamma_{f}$ to $\Gamma_{g}$, that is, $\rho$ is a bijective map from the set $\mathbf{F}_{2} \oplus F \oplus F$ of vertices of $\Gamma_{f}$ to the set $\mathbf{F}_{2} \oplus F \oplus F$ of vertices of $\Gamma_{g}$ such that vertices $(\varepsilon ; x, y)$ and $\left(\varepsilon^{\prime} ; x^{\prime}, y^{\prime}\right)\left(\varepsilon \in \mathbf{F}_{2}, x, y \in F\right)$ of $\Gamma_{f}$ are adjacent in $\Gamma_{f}$ if and only if $(\varepsilon ; x, y)^{\rho}$ and $\left(\varepsilon^{\prime} ; x^{\prime}, y^{\prime}\right)^{\rho}$ are adjacent in $\Gamma_{g}$. To distinguish the points and blocks of $\Gamma_{f}$ from those of $\Gamma_{g}$, we put suffixes $h$ ( $h=f$ or $g$ ) to the corresponding vectors or subsets of $\mathbf{F}_{2} \oplus F \oplus F$, when we regard them as vertices or subsets of vertices of $\Gamma_{h}$; for example,

$$
\begin{aligned}
& (\varepsilon ; x, y)=(\varepsilon ; x, y)_{f}=(\varepsilon ; x, y)_{g} \quad\left(\varepsilon \in \mathbf{F}_{2}, x, y \in F\right), \\
& \mathcal{P}_{f}=\mathcal{P}_{g}=\{(0 ; x, y) \mid x, y \in F\} \quad \text { and } \quad \mathcal{B}_{f}=\mathcal{B}_{g}=\{(1 ; x, y) \mid x, y \in F\} .
\end{aligned}
$$

Observe that $\rho$ is a map on $\mathbf{F}_{2} \oplus F \oplus F=\mathcal{P}_{f} \cup \mathcal{B}_{f}=\mathcal{P}_{g} \cup \mathcal{B}_{g}$.

We may assume that $\rho$ sends a point $(0 ; 0,0)_{f}$ of $\Gamma_{f}$ to a point $(0 ; 0,0)_{g}$ of $\Gamma_{g}$ because $\operatorname{Aut}\left(\Gamma_{g}\right)$ contains a group $\{\tau(a, b) \mid a, b \in F\}\langle\iota\rangle$ acting regularly on $\mathcal{P}_{g} \cup \mathcal{B}_{g}$ (see Lemma 4). Then $\rho$ is a map on $\mathbf{F}_{2} \oplus F \oplus F$ which sends the set $\mathcal{P}_{f}=\left\{(0 ; x, y)_{f} \mid\right.$ $x, y \in F\}$ (resp. $\mathcal{B}_{f}=\left\{(1 ; t, z)_{f} \mid t, z \in F\right\}$ ) of points (resp. blocks) of $\Gamma_{f}$ to the set $\mathcal{P}_{g}=\left\{(0 ; x, y)_{g} \mid x, y \in F\right\}$ (resp. $\left.\mathcal{B}_{g}=\left\{(1 ; t, z)_{g} \mid t, z \in F\right\}\right)$ of points (resp. blocks) of $\Gamma_{g}$. By Lemma 3, $\rho$ is $\mathbf{F}_{2}$-linear as a map on $\mathbf{F}_{2} \oplus F \oplus F$, regarded as a vector space over $\mathbf{F}_{2}$.

We use the letter $M_{f}$ (resp. $M_{g}$ ) to denote the stabilizer in $\operatorname{Aut}\left(\Gamma_{f}\right)$ (resp. $\left.\operatorname{Aut}\left(\Gamma_{g}\right)\right)$ of a point $(0 ; 0,0)_{f}\left(\operatorname{resp} .(0 ; 0,0)_{g}\right)$ (see Lemma 4). Since $\rho$ sends $(0 ; 0,0)_{f}$ to $(0 ; 0,0)_{g}$, we have $M_{g}=\rho^{-1} M_{f} \rho$. For a subgroup $G$ of $M_{f}$, we use the symbol $G^{\rho}$ to denote a subgroup $\rho^{-1} G \rho$ of $M_{g}$; namely, $G^{\rho}$ is the conjugate of $G$ under $\rho$.

The letters $T_{f}$ are $T_{g}$ are used to denote the groups of translations for $\Gamma_{f}$ and $\Gamma_{g}$, respectively (see (12) and (13)); namely, $T_{f}=\left\{t_{a} \mid a \in F\right\}$, where

$$
(0 ; x, y)_{f}^{t_{a}}=\left(0 ; x, y+B_{f}(x, a)\right)_{f},
$$

and

$$
(1 ; t, z)_{f}^{t_{a}}=\left(1 ; t+a, z+B_{f}(t, a)+\bar{f}(a)\right)_{f},
$$


for all $x, y \in F$. To distinguish the translations for $\Gamma_{g}$ from those for $\Gamma_{f}$, we use the letter $t_{a^{\prime}}^{\prime}$ to denote the translation for $\Gamma_{g}$ with respect to $a^{\prime} \in F$ :

$$
(0 ; x, y)_{g}^{t_{a^{\prime}}^{\prime}}=\left(0 ; x, y+B_{g}\left(x, a^{\prime}\right)\right)_{g}
$$

and

$$
(1 ; t, z)_{g}^{t_{a^{\prime}}^{\prime}}=\left(1 ; t+a^{\prime}, z+B_{g}\left(t, a^{\prime}\right)+\bar{g}\left(a^{\prime}\right)\right)_{g},
$$

for all $x, y \in F$. Remark that, in general, $T_{f}^{\rho}$ may not coincide with $T_{g}$.

Let $S_{f}$ be a Sylow 2-subgroup of the stabilizer $M_{f}$ containing the group $T_{f}$ of translations for $\Gamma_{f}$. Then $\rho^{-1} S_{f} \rho=S_{f}^{\rho}$ is a Sylow 2-subgroup of $M_{g}$. By the Sylow theorem, there is an element $\sigma$ of $M_{g}$ such that $\left(S_{f}^{\rho}\right)^{\sigma}$ contains $T_{g}$, the group of translations for $\Gamma_{g}$. Replacing $\rho$ by $\rho \sigma$, we may assume that $S_{f}^{\rho}$ contains $T_{g}$.

The image $(1 ; 0,0)_{f}^{\rho}$ of a block $(1 ; 0,0)_{f}$ of $\Gamma_{f}$ under $\rho$ is a block of $\Gamma_{g}$ adjacent to $(0 ; 0,0)_{f}^{\rho}=(0 ; 0,0)_{g}$. Since $T_{g}$ acts regularly on $\left\{(1 ; x, \bar{g}(x))_{g} \mid x \in F\right\}$ (see (22)), which is the set of blocks of $\Gamma_{g}$ adjacent to $(0 ; 0,0)_{g}$, we may assume that $(1 ; 0,0)^{\rho}=(1 ; 0,0)_{g}$, replacing $\rho$ by $\rho t_{a^{\prime}}^{\prime}$ for some $a^{\prime} \in F$.

Summarizing, we verified the following statement.

Lemma 10 Assume that $f$ and $g$ are quadratic APN functions on a field $F \cong \mathbf{F}_{2^{n}}$ with $n \geq 2$ which are CCZ-equivalent. Then there is a graph isomorphism $\rho$ from $\Gamma_{f}$ to $\Gamma_{g}$ which satisfies the following properties:

(i) $\rho$ is an $\mathbf{F}_{2}$-linear map on $\mathbf{F}_{2} \oplus F \oplus F$ preserving the hyperplane $\{(0 ; x, y) \mid$ $x, y \in F\}=\mathcal{P}_{f}=\mathcal{P}_{g}$. In particular, $(0 ; 0,0)_{f}^{\rho}=(0 ; 0,0)_{g}$.

(ii) $S_{f}^{\rho}$ is a Sylow 2-subgroup of $M_{g}$ containing $T_{g}$ for a Slyow 2-subgroup $S_{f}$ of $M_{f}$ containing $T_{f}$.

(iii) $(1 ; 0,0)_{f}^{\rho}=(1 ; 0,0)_{g}$.

In the following, we denote by $\rho$ a graph isomorphism from $\Gamma_{f}$ to $\Gamma_{g}$ which satisfies properties (i), (ii), and (iii) in Lemma 10.

A permutation $\pi$ on $F$. Now we introduce a permutation $\pi$ on $F$. Recall that the set $\left(\Gamma_{f}\right)_{1}\left((0 ; 0,0)_{f}\right)$ (resp. $\left.\left(\Gamma_{g}\right)_{1}\left((0 ; 0,0)_{g}\right)\right)$ of blocks of $\Gamma_{f}$ (resp. $\left.\Gamma_{g}\right)$ adjacent to $(0 ; 0,0)_{f}\left(\right.$ resp. $\left.(0 ; 0,0)_{g}\right)$ is given as $\left\{(1 ; x, \bar{f}(x))_{f} \mid x \in F\right\}$ (resp. $\left\{(1 ; x, \bar{g}(x))_{g} \mid\right.$ $x \in F\})$ by (7). Since $\rho$ maps the set $\left(\Gamma_{f}\right)_{1}\left((0 ; 0,0)_{f}\right)$ onto $\left(\Gamma_{g}\right)_{1}\left((0 ; 0,0)_{g}\right)$, there is a permutation $\pi$ on $F$ such that

$$
(1 ; x, \bar{f}(x))_{f}^{\rho}=\left(1 ; x^{\pi}, \bar{g}\left(x^{\pi}\right)\right)_{g} \quad \text { for all } x \in F .
$$

Since $(1 ; 0,0)_{f}^{\rho}=(1 ; 0,0)_{g}$, we have

$$
0^{\pi}=0 \text {. }
$$

Since $(0 ; x, \bar{f}(x))_{f}=(1 ; 0,0)_{f}+(1 ; x, \bar{f}(x))_{f}$, the linearity of $\rho$ and (23) imply

$$
(0 ; x, \bar{f}(x))_{f}^{\rho}=\left(0 ; x^{\pi}, \bar{g}\left(x^{\pi}\right)\right)_{g} \quad \text { for all } x \in F .
$$


Lemma 11 For all $x, y \in F$, we have

$$
\left(0 ; 0, B_{f}(x, y)\right)_{f}^{\rho}=\left(0 ;(x+y)^{\pi}+x^{\pi}+y^{\pi}, \bar{g}\left((x+y)^{\pi}\right)+\bar{g}\left(x^{\pi}\right)+\bar{g}\left(y^{\pi}\right)\right)_{g} .
$$

Proof Since

$$
\left(0 ; 0, B_{f}(x, y)\right)_{f}=(0 ; x, \bar{f}(x))_{f}+(0 ; y, \bar{f}(y))_{f}+(0 ; x+y, \bar{f}(x+y))_{f},
$$

the linearity of $\rho$ and (25) imply

$$
\begin{aligned}
& \left(0 ; 0, B_{f}(x, y)\right)_{f}^{\rho} \\
& \quad=(0 ; x, \bar{f}(x))_{f}^{\rho}+(0 ; y, \bar{f}(y))_{f}^{\rho}+(0 ; x+y, \bar{f}(x+y))_{f}^{\rho} \\
& \quad=\left(0 ; x^{\pi}, \bar{g}\left(x^{\pi}\right)\right)_{g}+\left(0 ; y^{\pi}, \bar{g}\left(y^{\pi}\right)\right)_{g}+\left(0 ;(x+y)^{\pi}, \bar{g}\left((x+y)^{\pi}\right)\right)_{g} \\
& \quad=\left(0 ;(x+y)^{\pi}+x^{\pi}+y^{\pi}, \bar{g}\left((x+y)^{\pi}\right)+\bar{g}\left(x^{\pi}\right)+\bar{g}\left(y^{\pi}\right)\right)_{g} .
\end{aligned}
$$

Thus, (26) is verified.

Conditions (a) and (b) In what follows, we consider the following conditions:

(a) $\rho$ is a graph isomorphism from $\Gamma_{f}$ to $\Gamma_{g}$ satisfying properties (i), (ii), and (iii) in Lemma 10.

(b) $\rho$ does not send $\left\{(0 ; 0, y)_{f} \mid y \in F\right\}$ to $\left\{(0 ; 0, y)_{g} \mid y \in F\right\}$.

(b') $f$ is not EA-equivalent to $g$.

As we will see below, to assume (a) and (b) is equivalent to assume (a) and ( $\left.b^{\prime}\right)$.

Lemma 12 Under condition (a), the map $\rho$ sends $\left\{(0 ; 0, y)_{f} \mid y \in F\right\}$ to $\left\{(0 ; 0, y)_{g} \mid\right.$ $y \in F\}$ if and only if it induces an EA-equivalence of $f$ with $g$.

Proof If $\rho$ sends $\left\{(0 ; 0, y)_{f} \mid y \in F\right\}=Y$ to $\left\{(0 ; 0, y)_{g} \mid y \in F\right\}=Y, \rho$ is represented by $\mathbf{F}_{2}$-linear bijective maps $\alpha$ and $\delta$ on $F$ and an $\mathbf{F}_{2}$-linear map $\beta$ on $F$ such that $(0 ; x, y)_{f}^{\rho}=\left(0 ; x^{\alpha}, x^{\beta}+y^{\delta}\right)_{g}$. Then we have $\left(0 ; x^{\pi}, \bar{g}\left(x^{\pi}\right)\right)_{g}=(0 ; x, \bar{f}(x))_{f}^{\rho}=$ $\left(0 ; x^{\alpha}, x^{\beta}+\bar{f}(x)^{\delta}\right)_{g}$ from (23). Thus, $\pi=\alpha$ is an $\mathbf{F}_{2}$-linear bijection on $F$, and $\bar{g}\left(x^{\alpha}\right)=x^{\beta}+\bar{f}(x)^{\delta}$. This shows that $g\left(x^{\alpha}\right)=f(x)^{\delta}+\left(x^{\beta}+g(0)+f(0)^{\delta}\right)$ for all $x \in F$, whence $f$ is EA-equivalent to $g$. The converse immediately follows from Definition 1 (EA).

In the remainder of this paper, we will derive a contradiction, assuming that $\rho$ satisfies conditions (a) and (b) above. This implies that $\rho$ with condition (a) should not satisfy $\left(\mathrm{b}^{\prime}\right)$ by Lemma 12; namely, it should induce an EA-equivalence of $f$ with $g$. This establishes Theorem 1.

Lemma 13 Under assumptions (a) and (b) above, the following hold.

(1) The center $Z\left(S_{f}\right)$ of a Sylow 2-subgroup $S_{f}$ is of order 2 generated by a nonidentity translation $t_{a}\left(a \in F^{\times}\right)$for $\Gamma_{f}$. 
(2) The center $Z\left(S_{f}\right)^{\rho}$ of a Sylow 2-subgroup $S_{f}^{\rho}$ is a group of order 2 generated by a nonidentity translation $t_{a^{\prime}}^{\prime}\left(a^{\prime} \in F^{\times}\right)$for $\Gamma_{g}$.

(3) We have $t_{a^{\prime}}^{\prime}=\left(t_{a}\right)^{\rho}$ and $a^{\prime}=a^{\pi}$.

Proof Suppose that the center $Z\left(S_{f}\right)$ has order greater than 2. By Lemma 9 applied to $S_{f}$, the center $Z\left(S_{f}\right)$ contains at least two distinct nonidentity translations $t_{a}$ and $t_{b}$ for some distinct $a, b \in F^{\times}$. Then from Lemma 6(3) it follows that the centralizer $C_{\mathcal{P}_{f}}\left(Z\left(S_{f}\right)\right)$ of $Z\left(S_{f}\right)$ on $\mathcal{P}_{f}$ coincides with $\left\{(0 ; 0, y)_{f} \mid y \in F\right\}=$ $C_{\mathcal{P}_{f}}\left(t_{a}\right) \cap C_{\mathcal{P}_{f}}\left(t_{b}\right)$. (Observe that $(0 ; 0, y)_{f}$ for any $y \in F$ is fixed by any other translation $t_{c}$ in $Z\left(S_{f}\right)$.)

The conjugate $Z\left(S_{f}\right)^{\rho}$ of $Z\left(S_{f}\right)$ by $\rho$ is the center $Z\left(S_{f}^{\rho}\right)$ of a Sylow 2-subgroup $S_{f}^{\rho}$, which contains the group $T_{g}$ of translations for $\Gamma_{g}$ by condition (ii) of Lemma 10. Since $Z\left(S_{f}^{\rho}\right)$ has the same order as $Z\left(S_{f}\right)$, it follows from Lemma 9 applied to $S_{f}^{\rho}$ (a Sylow subgroup of $M_{g}$ with notation in Lemma 4) that $Z\left(S_{f}^{\rho}\right)$ contains at least two distinct nonidentity translations $t_{a^{\prime}}^{\prime}$ and $t_{b^{\prime}}^{\prime}$. Thus, the centralizer $C_{\mathcal{P}_{g}}\left(Z\left(S_{f}^{\rho}\right)\right)$ of $Z\left(S_{f}^{\rho}\right)$ on $\mathcal{P}_{g}$ coincides with $\left\{(0 ; 0, y)_{g} \mid y \in F\right\}=C_{\mathcal{P}_{g}}\left(t_{a^{\prime}}^{\prime}\right) \cap C_{\mathcal{P}_{g}}\left(t_{b^{\prime}}^{\prime}\right)$ by Lemma 6(3) applied to $\Gamma_{g}$.

Since $\rho$ sends $C_{\mathcal{P}_{f}}\left(Z\left(S_{f}\right)\right)$ to $C_{\mathcal{P}_{g}}\left(Z\left(S_{f}^{\rho}\right)\right)$, this contradicts our assumption that $Y$ is not stabilized by $\rho$ (condition (b)). Hence, we conclude that $Z\left(S_{f}\right)$ has order 2. Since $Z\left(S_{f}\right) \subset T_{f}$ by Lemma 9 , there is a nonzero element $a$ of $F$ such that the translation $t_{a}$ generates $Z\left(S_{f}\right)$. This proves Claim (1).

Since $Z\left(S_{f}^{\rho}\right)$ is the conjugate of $Z\left(S_{f}\right)$, it also has order 2. By Lemma 9 applied to $S_{f}^{\rho}, Z\left(S_{f}^{\rho}\right)$ is generated by a nonidentity translation $t_{a^{\prime}}^{\prime}$ in $T_{g}$. This shows Claim (2).

From Claims (1) and (2) we have $\left(t_{a}\right)^{\rho}=t_{a^{\prime}}^{\prime}$, Then the action of $t_{a^{\prime}}^{\prime}$ to the block $(1 ; 0,0)_{g}$ is calculated as follows, using (20), (22), and (23):

$$
\begin{aligned}
\left(1 ; a^{\prime}, \bar{g}\left(a^{\prime}\right)\right)_{g} & =(1 ; 0,0)_{g}^{t_{a^{\prime}}^{\prime}}=(1 ; 0,0)_{g}^{\rho^{-1} t_{a} \rho}= \\
& =(1 ; 0,0)_{f}^{t_{a} \rho}=(1 ; a, \bar{f}(a))_{f}^{\rho}=\left(1 ; a^{\pi}, \bar{g}\left(a^{\pi}\right)\right) .
\end{aligned}
$$

Thus, we have $a^{\prime}=a^{\pi}$. This verifies Claim (3).

Notation In the following, we use the letters $a$ and $a^{\prime}$ to denote nonzero elements $a$ and $a^{\prime}$ of $F$ such that $Z\left(S_{f}\right)=\left\langle t_{a}\right\rangle$ and $Z\left(S_{f}^{\rho}\right)=\left\langle t_{a^{\prime}}^{\prime}\right\rangle$ (see Lemma 13).

We also use the letter $\alpha$ to denote $\alpha(a)$ defined in (11); namely, $\alpha$ denotes the specific nonzero element of $F$ for which

$$
H_{a}=\left\{B_{f}(a, x) \mid x \in F\right\}=\{y \in F \mid \operatorname{Tr}(\alpha y)=0\} .
$$

We also set

$$
H_{a^{\prime}}^{\prime}:=\left\{B_{g}\left(a^{\prime}, x\right) \mid x \in F\right\},
$$

the hyperplane of $F$ determined by $a^{\prime}$ and a quadratic APN function $g$. 
Lemma 14 Assuming conditions (a) and (b) above, we have:

$$
\begin{aligned}
& \left\{(0 ; 0, y)_{f} \mid y \in H_{a}\right\}^{\rho}=\left\{\left(0 ; 0, y^{\prime}\right)_{g} \mid y^{\prime} \in H_{a^{\prime}}^{\prime}\right\}, \\
& \left\{(0 ; 0, y)_{f} \mid y \in F\right\}^{\rho}=\left\{\left(0 ; a^{\prime},\left(\varepsilon^{\prime}+1\right) c^{\prime}+y^{\prime}\right),\left(0 ; 0, y^{\prime}\right) \mid y^{\prime} \in H_{a^{\prime}}^{\prime}\right\},
\end{aligned}
$$

where $c^{\prime}$ is a fixed element in $F \backslash H_{a^{\prime}}^{\prime}$, and $\varepsilon^{\prime}$ is 0 or 1 , according to $\bar{g}\left(a^{\prime}\right) \in H_{a^{\prime}}^{\prime}$ or not.

Proof The commutator subspace $\left[\mathcal{P}_{f}, Z\left(S_{f}\right)\right]=\left[\mathcal{P}_{f}, t_{a}\right]=\left\{(0 ; 0, y)_{f} \mid y \in H_{a}\right\}$ of $Z\left(S_{f}\right)$ on $\mathcal{P}_{f}$ (see Lemma 6(2) for $t_{a}$ ) is sent by $\rho$ to the commutator subspace $\left[\mathcal{P}_{g}, Z(S)^{\rho}\right]=\left[\mathcal{P}_{g}, t_{a^{\prime}}^{\prime}\right]=\left\{\left(0 ; 0, y^{\prime}\right)_{g} \mid y^{\prime} \in H_{a^{\prime}}^{\prime}\right\}$ of $Z\left(S_{f}\right)^{\rho}$ on $\mathcal{P}_{g}$ (see Lemma 6(2) for $t_{a^{\prime}}^{\prime}$ ). This shows the first claim, (27), of the lemma.

Next consider the subspace $Y_{f}:=\left\{(0 ; 0, y)_{f} \mid y \in F\right\}$ of $\mathbf{F}_{2} \oplus F \oplus F$. Observe that this is one of the two $n$-dimensional subspaces of $C_{\mathcal{P}_{f}}\left(t_{a}\right)$ satisfying conditions in Lemma 7; namely, it contains $\left[\mathcal{P}_{f}, t_{a}\right]=\left\{(0 ; 0, y)_{f} \mid y \in H_{a}\right\}$ but does not contain any point at distance two from $(0 ; 0,0)_{f}$. Remark that the image $Y_{f}^{\rho}$ of $Y_{f}$ under $\rho$ is an $n$-dimensional subspace of $C_{\mathcal{P}_{g}}\left(t_{a^{\prime}}^{\prime}\right)=C_{\mathcal{P}_{f}}\left(t_{a}\right)^{\rho}$ containing $\left[\mathcal{P}_{f}, t_{a}\right]^{\rho}=\left\{\left(0 ; 0, y^{\prime}\right)_{g} \mid y^{\prime} \in H_{a^{\prime}}^{\prime}\right\}$ (see the first claim of the lemma) but having no point at distance two from $(0 ; 0,0)_{g}=(0 ; 0,0)_{f}^{\rho}$. Hence, applying Lemma 7 to $t_{a^{\prime}}^{\prime}$ and $Y_{f}^{\rho}$, we have either $Y_{f}^{\rho}=Y_{g}=\left\{(0 ; 0, y)_{g} \mid y \in F\right\}$ or $Y_{f}^{\rho}=Y_{g}\left(a^{\prime}\right)=$ $\left\{\left(0 ; a^{\prime},\left(\varepsilon^{\prime}+1\right) c^{\prime}+y^{\prime}\right),\left(0 ; 0, y^{\prime}\right) \mid y^{\prime} \in H_{a^{\prime}}^{\prime}\right\}$, where $c^{\prime}$ is a fixed element of $F \backslash H_{a^{\prime}}^{\prime}$, and $\varepsilon^{\prime}=0$ or 1 according to $\bar{g}\left(a^{\prime}\right) \in H_{a^{\prime}}^{\prime}$ or not. If the former case holds, the map $\rho$ preserves $\{(0 ; 0, y) \mid y \in F\}=Y_{f}=Y_{g}^{\prime}$, which contradicts condition (b). Hence, the latter holds, which verifies the second claim, (28), of the lemma.

In view of Lemma 14 , a point $(0 ; 0, z)_{f}$ of $\Gamma_{f}$ is mapped by $\rho$ to a point of the form $\left(0 ; 0, z^{\prime}\right)_{g}$ or $\left(0 ; a^{\prime}, z^{\prime \prime}\right)_{g}$ according to $z \in H_{a}$ or $z \notin H_{a}$. Namely, the second component of $(0 ; 0, z)_{f}^{\rho}$ is 0 or $a^{\prime}$ according to $z \in H_{a}$ or not.

Using the element $\alpha$ defined in Notation, this observation is rephrased as follows: for any $z \in F$, the second component of $(0 ; 0, z)_{f}^{\rho}$ coincides with $\operatorname{Tr}(\alpha z) a^{\prime}$. Applying this observation to a point $(0 ; 0, z)_{f}$ with $z$ in the form $B_{f}(x, y)$ for some $x, y \in F$, we have the following lemma from (26).

Lemma 15 Assume conditions (a) and (b) above. For any $x, y \in F$, we have

$$
(x+y)^{\pi}+x^{\pi}+y^{\pi}=\kappa(x, y) a^{\prime},
$$

where $\kappa$ is the map from $F \times F$ to $\mathbf{F}_{2}$ defined by

$$
\kappa(x, y):=\operatorname{Tr}\left(\alpha B_{f}(x, y)\right) .
$$

We give some remarks on the map $\kappa$ defined in Lemma 15. Since $f$ is quadratic, $B_{f}$ is bilinear on $F$. Hence, $\kappa$ is a bilinear form on $F$. Since $B_{f}(x, x)=0, \kappa$ is alternating; $\kappa(x, x)=0$ for all $x \in F$ and hence symmetric: $\kappa(x, y)=\kappa(y, x)$ for all $x, y \in F$. From Definition (30) and Definition (11) we have that $\kappa(x, y)=0$ if and only if $B_{f}(x, y)$ lies in the hyperplane $H_{a}$ of $F$. 
Lemma 16 Assume conditions (a) and (b) above. If $\kappa(x, y)=0$ for $x, y \in F$, we have

$$
\left(0 ; 0, B_{f}(x, y)\right)_{f}^{\rho}=\left(0 ; 0, B_{g}\left(x^{\pi}, y^{\pi}\right)\right)_{g} .
$$

Proof If $\kappa(x, y)=0$, then we have $(x+y)^{\pi}=x^{\pi}+y^{\pi}$ from (29). Then the third component of $\left(0 ; 0, B_{f}(x, y)\right)_{f}^{\rho}$, which is given as $\bar{g}\left((x+y)^{\pi}\right)+\bar{g}\left(x^{\pi}\right)+\bar{g}\left(y^{\pi}\right)$ by (26), coincides with $\bar{g}\left(x^{\pi}+y^{\pi}\right)+\bar{g}\left(x^{\pi}\right)+\bar{g}\left(y^{\pi}\right)=B_{g}\left(x^{\pi}, y^{\pi}\right)$. This verifies the lemma.

The above lemma imposes a strong restriction on the alternating form $\kappa$.

Lemma 17 Assume conditions (a) and (b) above. Let $y$ be any element in $F \backslash\{0, a\}$. Then the subspace $y^{\perp}:=\{x \in F \mid \kappa(y, x)=0\}$ of $F$ orthogonal to $y$ with respect to $\kappa$ is totally isotropic; namely, $\kappa\left(x_{1}, x_{2}\right)=0$ for any $x_{1}, x_{2} \in y^{\perp}$.

Proof Let $y$ be any element in $F \backslash\{0, a\}$. Take any element $x_{i}$ in $y^{\perp}(i=1,2)$. Since $\kappa\left(x_{i}, y\right)=0$ for $i=1,2$ and $\kappa$ is bilinear, we have $\kappa\left(x_{1}+x_{2}, y\right)=0$. Then it follows from (31) that

$$
\left(0 ; 0, B_{f}\left(x_{1}+x_{2}, y\right)\right)_{f}^{\rho}=\left(0 ; 0, B_{g}\left(\left(x_{1}+x_{2}\right)^{\pi}, y^{\pi}\right)\right)_{g} .
$$

Since $\rho$ is linear and $B_{f}$ and $B_{g}$ are bilinear, the left-hand side of this equation can be written as follows, using (31) and the assumption that $\kappa\left(x_{i}, y\right)=0, i=1,2$ :

$$
\begin{aligned}
& \left(0 ; 0, B_{f}\left(x_{1}, y\right)\right)_{f}^{\rho}+\left(0 ; 0, B_{f}\left(x_{2}, y\right)\right)_{f}^{\rho} \\
& \quad=\left(0 ; 0, B_{g}\left(x_{1}^{\pi}, y^{\pi}\right)\right)_{g}+\left(0 ; 0, B_{g}\left(x_{2}^{\pi}, y^{\pi}\right)\right)_{g} \\
& \quad=\left(0 ; 0, B_{g}\left(x_{1}^{\pi}+x_{2}^{\pi}, y^{\pi}\right)\right)_{g} .
\end{aligned}
$$

Thus, comparing the third components, we have $B_{g}\left(\left(x_{1}+x_{2}\right)^{\pi}, y^{\pi}\right)=B_{g}\left(x_{1}^{\pi}+\right.$ $\left.x_{2}^{\pi}, y^{\pi}\right)$. From the bilinearity of $B_{g}$ we then have

$$
B_{g}\left(\left(x_{1}+x_{2}\right)^{\pi}+x_{1}^{\pi}+x_{2}^{\pi}, y^{\pi}\right)=0 .
$$

Applying (29), this implies that

$$
B_{g}\left(\kappa\left(x_{1}, x_{2}\right) a^{\prime}, y^{\pi}\right)=0 .
$$

Since $\kappa\left(x_{1}, x_{2}\right)$ is an element of $\mathbf{F}_{2}$, we then have

$$
\kappa\left(x_{1}, x_{2}\right) B_{g}\left(a^{\prime}, y^{\pi}\right)=0 .
$$

Now $B_{g}\left(a^{\prime}, y^{\pi}\right)=0$ if and only if $y^{\pi}=0$ or $y^{\pi}=a^{\prime}$. By (24) and Lemma 13(3), this is equivalent to $y=0$ or $y=a$. Thus, by our choice of $y$, we should have $\kappa\left(x_{1}, x_{2}\right)=0$. Since this holds for every $x_{1}, x_{2}$ in $y^{\perp}$, the subspace $y^{\perp}$ is totally isotropic. 
Proof of Theorem 1 Now we obtain a final contradiction. We assume conditions (a) and (b) above.

Since $\kappa$ is an alternating form on $F, F$ is decomposed as follows from the standard theory on alternating bilinear forms:

$$
F=R \oplus\left\langle e_{1}, \ldots, e_{r}\right\rangle \oplus\left\langle f_{1}, \ldots, f_{r}\right\rangle
$$

where $R=\{x \in F \mid \kappa(x, y)=0(\forall y \in F)\}$ is the radical, and $\left\{e_{i}, f_{i}\right\}(i=1, \ldots, r)$ are parabolic pairs; namely, $\kappa\left(e_{i}, e_{j}\right)=0=\kappa\left(f_{i}, f_{j}\right)$ and $\kappa\left(e_{i}, f_{j}\right)=\delta_{i, j}$ for all $i, j \in\{1, \ldots, r\}$.

Assume that $r \geq 2$. Then the subspaces $e_{1}^{\perp}$ and $f_{1}^{\perp}$ contain $\left\langle e_{2}, f_{2}\right\rangle$, and therefore none of them is totally isotropic. This contradicts Lemma 17, because it states that $a$ is a unique possible nonzero element $y$ in $F$ such that $y^{\perp}$ is not totally isotropic. Thus, we have $r \leq 1$.

Assume that $r=1$. Then the radical $R$ has codimension 2 in $F$. As we assume that $f$ and $g$ are CCZ-equivalent but EA-inequivalent APN functions on $F \cong \mathbf{F}_{2^{n}}$, we must have $n \geq 4$. Then $R$ has dimension at least 2, and hence $R$ contains a nonzero element $b$ distinct from $a$. However, $b^{\perp}=F$ is not totally isotropic, which contradicts Lemma 17.

Hence, we have $r=0$, namely, $F$ itself is totally isotropic with respect to $\kappa$. However, this implies that $B_{f}(x, y)$ lies in a hyperplane $H_{a}$ of $F$ for every $x, y \in F$. This contradicts Lemma 5.

Thus, we have a final contradiction, and Theorem 1 is established.

\section{References}

1. Bracken, C., Byrne, E., McGuire, G., Nebe, G.: On equivalence of quadratic APN-functions. Des. Codes Cryptogr. 61, 261-272 (2010). doi:10.1007/s10623-010-9475-8

2. Yoshiara, S.: Dimensional dual hyperovals associated with quadratic APN functions. Innov. Incid. Geom. 8, 147-169 (2008)

3. Yoshiara, S.: Notes on APN functions, semibiplanes and dimensional dual hyperovals. Des. Codes Cryptogr. 56, 197-218 (2010) 